\title{
Extended Alamouti Codes in Correlated Channels Using Partial Feedback
}

\author{
Biljana Badic, Markus Rupp, Hans Weinrichter \\ Institute for Communications and Radio Frequency Engineering \\ Vienna University of Technology \\ Gusshausstr.25, A-1040 Vienna, Austria \\ (bbadic, mrupp, jweinri)@nt.tuwien.ac.at
}

\begin{abstract}
In this paper we present a family of quasiorthogonal space-time codes for correlated MIMO (multiinput/multi-output) channels using four and eight transmit antennas when only partial channel state information is sent back to the transmitter. Transmit diversity is improved up to nearly the optimum diversity value. This high diversity can be exploited with zero forcing receivers as well as with maximum-likelihood receivers.
\end{abstract}

\section{INTRODUCTION}

Since the pioneering work of Alamouti [1], several orthogonal and quasi-orthogonal space-time block codes (STBCs) have been investigated. It has been shown in [2], that orthogonal full-rate design offering full diversity for any arbitrary complex symbol constellation is limited to the case of two transmit antennas. Either diversity, data rate or decoding complexity must be sacrificed if the number of transmit antennas is increased. To preserve the full symbol rate, however with a small loss in performance, quasi-orthogonal designs have been proposed [3]. Exploiting channel state information (CSI) at the transmitter and at the receiver the bit error ratio (BER) and outage performance is improved compared to the case when only the receiver has perfect channel knowledge. In some schemes, low decoding complexity, high diversity and a higher code rate can be obtained even if only partial channel information is sent back to the transmitter. An example of this is given in [4] where the the space time block code is combined with limited feedback to take advantages of it.

Research on adapting the block code to partial feedback has been an intensive area of research [5]-[7]. However, mostly channel models with independent and identical distribution (i.i.d.) have been used. While this is far from practical setups, the advantage of such simplification is that much of the performance can be predicted in closed form mathematical expressions.

In this paper, STBCs are investigated under correlated channels as can be expected due to the nature of transmit and receive antennas and the wireless environment. Since for general MIMO transmission closed form expressions are available only for limited cases [8], our work concentrates on simulations. In particular, we utilize the so called Kronecker channel model [9] and extended Alamouti STBCs, designed for four and eight transmit antennas. First we present a transmission system where only one channel information bit $b$ per code block is returned to the transmitter. Depending on the value of $b$ the transmitter switches between two predefined STBCs and chooses that code matrix which achieves better error performance. In a second approach, sending back two information bits per code block to the transmitter, the transmitter can switch between four predefined space-time block codes achieving still lower BER, nearly to optimum.

The paper is organized as follows. First, an overview of the Extended Alamouti space-time block codes (EACs) for four and eight transmit antennas is given. Section III describes channel adaptive EACs and transmission schemes using one or two bits fed back per code block. Simulation results for correlated channels are presented in Section IV and conclusions are summarized in Section V.

\section{EXtended Alamouti Codes FOR FOUR AND EIGHT TRANSMIT ANTENNAS}

\section{A. Extended Alamouti Scheme for four transmit antennas}

In the following the extended Alamouti Scheme for four and eight antennas is explained. In the case of four transmit antennas, the well-known Alamouti Code [1]

$$
\mathbf{S}^{(2)}=\left[\begin{array}{rr}
s_{1} & s_{2} \\
s_{2}^{*} & -s_{1}^{*}
\end{array}\right]
$$

can be used as a building block to obtain a $(4 \times 4)$ code block given in Equ.(2) [11]:

$\mathbf{S}^{(4)}=\mathbf{S}_{1}^{(4)}=\left[\begin{array}{ll}\mathbf{S}_{1}^{(2)} & \mathbf{S}_{2}^{(2)} \\ \mathbf{S}_{2}^{(2) *} & -\mathbf{S}_{1}^{(2) *}\end{array}\right]=\left[\begin{array}{rrrr}s_{1} & s_{2} & s_{3} & s_{4} \\ s_{2}^{*} & -s_{1}^{*} & s_{4}^{*} & -s_{3}^{*} \\ s_{3}^{*} & s_{4}^{*} & -s_{1}^{*} & -s_{2}^{*} \\ s_{4} & -s_{3} & -s_{2} & s_{1}\end{array}\right]$

$\mathbf{S}_{1}^{(2)}$ and $\mathbf{S}_{2}^{(2)}$ correspond to $(2 \times 2)$ code blocks defined in (1). Using one receive antenna during 4 successive time slots an equivalent $(4 \times 4)$ virtual structured channel matrix $\mathbf{H}_{v 1}^{(4)}$ results as:

$$
\mathbf{H}_{v 1}^{(4)}=\left[\begin{array}{cc}
\mathbf{H}_{v 1}^{(2)} & \mathbf{H}_{v 2}^{(2)} \\
-\mathbf{H}_{v 2}^{(2) *} & \mathbf{H}_{v 1}^{(2) *}
\end{array}\right]=\left[\begin{array}{rrrr}
h_{1} & h_{2} & h_{3} & h_{4} \\
-h_{2}^{*} & h_{1}^{*} & -h_{4}^{*} & h_{3}^{*} \\
-h_{3}^{*} & -h_{4}^{*} & h_{1}^{*} & h_{2}^{*} \\
h_{4} & -h_{3} & -h_{2} & h_{1}
\end{array}\right]
$$

where

$$
\mathbf{H}_{v}^{(2)}=\left[\begin{array}{rr}
h_{1} & h_{2} \\
-h_{2}^{*} & h_{1}^{*}
\end{array}\right]
$$

is an orthogonal, $(2 \times 2)$ sub matrix. In this way, the extended Alamouti Scheme simulates a MIMO system with a highly structured virtual $(4 \times 4)$ MIMO channel. 


\section{B. Extended Alamouti Scheme for eight transmit antennas}

Extending the Alamouti signalling scheme given in Equ.(2) once more, an EAC for eight transmit antennas can be obtained. The EAC for eight transmit antennas is now defined as [10]:

$$
\begin{aligned}
& \mathbf{S}^{(8)}=\left[\begin{array}{ll}
\mathbf{S}_{1}^{(4)} & \mathbf{S}_{5}^{(4)} \\
\mathbf{S}_{5}^{(4) *} & -\mathbf{S}_{1}^{(4) *}
\end{array}\right]= \\
& {\left[\begin{array}{rrrr|rrrr}
s_{1} & s_{2} & s_{3} & s_{4} & s_{5} & s_{6} & s_{7} & s_{8} \\
s_{2}^{*} & -s_{1}^{*} & s_{4}^{*} & -s_{3}^{*} & s_{6}^{*} & -s_{5}^{*} & s_{8}^{*} & -s_{7}^{*} \\
s_{3}^{*} & s_{4}^{*} & -s_{1}^{*} & -s_{2}^{*} & s_{7}^{*} & s_{8}^{*} & -s_{5}^{*} & -s_{6}^{*} \\
s_{4} & -s_{3} & -s_{2} & s_{1} & s_{8} & -s_{7} & -s_{6} & s_{5} \\
\hline s_{5}^{*} & s_{6}^{*} & s_{7}^{*} & s_{8}^{*} & -s_{1}^{*} & -s_{2}^{*} & -s_{3}^{*} & -s_{4}^{*} \\
s_{6} & -s_{5} & s_{8} & -s_{7} & -s_{2} & s_{1} & -s_{4} & s_{3} \\
s_{7} & s_{8} & -s_{5} & -s_{6} & -s_{3} & -s_{4} & s_{1} & s_{2} \\
s_{8}^{*} & -s_{7}^{*} & -s_{6}^{*} & s_{5}^{*} & -s_{4}^{*} & s_{3}^{*} & s_{2}^{*} & -s_{1}^{*}
\end{array}\right],}
\end{aligned}
$$

where we have defined a new block of four symbols $\mathbf{S}_{5}^{(4)}$ containing the symbols $\left\{s_{5}, s_{6}, s_{7}, s_{8}\right\}$. With this code block eight information symbols and their conjugates are transmitted in various combinations over the eight transmit antennas in eight successive time slots. As explained before, the system can be equivalently described as an $(8 \times 8)$ MIMO system with an $(8 \times 8)$ virtual channel matrix $\mathbf{H}_{v}^{(8)}$ resulting in :

$$
\begin{gathered}
\mathbf{H}_{v}^{(8)}=\left[\begin{array}{crrrrr}
\mathbf{H}_{v 1}^{(4)} & \mathbf{H}_{v 5}^{(4)} \\
-\mathbf{H}_{v 5}^{(4) *} & \mathbf{H}_{v 1}^{(4) *}
\end{array}\right]= \\
{\left[\begin{array}{rrrrrrrr}
h_{1} & h_{2} & h_{3} & h_{4} & h_{5} & h_{6} & h_{7} & h_{8} \\
-h_{2}^{*} & h_{1}^{*} & -h_{4}^{*} & h_{3}^{*} & -h_{6}^{*} & h_{5}^{*} & -h_{8}^{*} & h_{7}^{*} \\
-h_{3}^{*} & -h_{4}^{*} & h_{1}^{*} & h_{2}^{*} & -h_{7}^{*} & -h_{8}^{*} & h_{5}^{*} & h_{6}^{*} \\
h_{4} & -h_{3} & -h_{2} & h_{1} & h_{8} & -h_{7} & -h_{6} & h_{5} \\
\hline-h_{5}^{*} & -h_{6}^{*} & -h_{7}^{*} & -h_{8}^{*} & h_{1}^{*} & h_{2}^{*} & h_{3}^{*} & h_{4}^{*} \\
h_{6} & -h_{5} & h_{8} & -h_{7} & -h_{2} & h_{1} & -h_{4} & h_{3} \\
h_{7} & h_{8} & -h_{5} & -h_{6} & -h_{3} & -h_{4} & h_{1} & h_{2} \\
-h_{8}^{*} & h_{7}^{*} & h_{6}^{*} & -h_{5}^{*} & h_{4}^{*} & -h_{3}^{*} & -h_{2}^{*} & h_{1}^{*}
\end{array}\right] .}
\end{gathered}
$$

Unfortunately, both virtual channel matrices $\mathbf{H}_{v}^{(4)}$ and $\mathbf{H}_{v}^{(8)}$ are no more orthogonal. However, in the following it will be shown that $\mathbf{H}_{v}^{(4)}$ and $\mathbf{H}_{v}^{(8)}$ are "nearly orthogonal".

\section{Channel Adaptive Extended Alamouti CODES(CAEACS)}

\section{A. CAEACs using one feedback bit per code block}

Here CAEACs for four transmit antennas using a family of two EACs are introduced. The corresponding transmission scheme is shown in Fig. 1. The transmitter selects one of two very similar EACs $\mathbf{S}_{1}^{(4)}$ or $\mathbf{S}_{2}^{(4)}$ to transmit the signal vector $\mathbf{s}$ over the real channel $\mathbf{h}$. The selection algorithm is controlled by the receiver, that tells the transmitter which one of the two EACs will achieve a more reliable transmission. At the receiver this knowledge is gained from the knowledge of the channel transfer vector $\mathbf{h}$ (we assume that the receiver has perfect channel knowledge). The family of EACs consists of two $(4 \times 4)$ code blocks $\mathbf{S}_{1}^{(4)}$ and $\mathbf{S}_{2}^{(4)}$ corresponding to four transmit antennas and $T=4$ time intervals. The channel transfer vector $\mathbf{h}=\left[h_{1}, h_{2}, h_{3}, h_{4}\right]^{T}$ may fade in

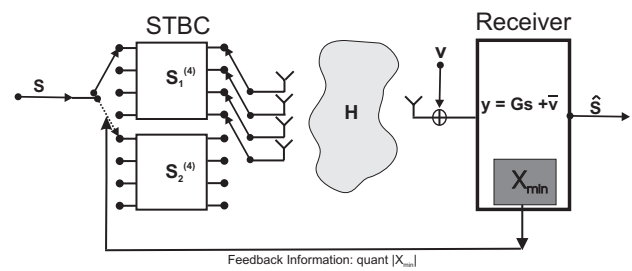

Fig. 1. Scheme with One Feedback Bit per Code Block and Four Transmit Antennas

any arbitrary way but it is assumed that $\mathbf{h}$ remains constant during the code block of length $\mathrm{T}$. The signal transmission can be described by

$$
\mathbf{y}_{i}=\mathbf{S}_{i}^{(4)} \mathbf{h}+\mathbf{v} ; \quad i=1,2,
$$

where $\mathbf{y}_{i}$ is the $(4 \times 1)$ vector of received signals. $\mathbf{S}_{i}^{(4)}$ is either $\mathbf{S}_{1}^{(4)}$ or $\mathbf{S}_{2}^{(4)}$ depending on the channel parameter $b$, defined below, which is fed back from the receiver. $\mathbf{v}$ is the $(4 \times 1)$ noise vector with complex Gaussian components with zero mean and variance $\sigma_{v}^{2}$. $\mathbf{S}_{1}^{(4)}$ and $\mathbf{S}_{2}^{(4)}$ are defined as:

$$
\begin{aligned}
\mathbf{S}_{1}^{(4)} & =\left[\begin{array}{rrrr}
s_{1} & s_{2} & s_{3} & s_{4} \\
s_{2}^{*} & -s_{1}^{*} & s_{4}^{*} & -s_{3}^{*} \\
s_{3}^{*} & s_{4}^{*} & -s_{1}^{*} & -s_{2}^{*} \\
s_{4} & -s_{3} & -s_{2} & s_{1}
\end{array}\right] ; \\
\mathbf{S}_{2}^{(4)} & =\left[\begin{array}{rrrr}
-s_{1} & s_{2} & s_{3} & s_{4} \\
-s_{2}^{*} & -s_{1}^{*} & s_{4}^{*} & -s_{3}^{*} \\
-s_{3}^{*} & s_{4}^{*} & -s_{1}^{*} & -s_{2}^{*} \\
-s_{4} & -s_{3} & -s_{2} & s_{1}
\end{array}\right] .
\end{aligned}
$$

Equ.(7) can be rewritten as

$$
\mathbf{y}_{i}=\mathbf{H}_{v i}^{(4)} \mathbf{s}+\mathbf{v} ; \quad i=1,2,
$$

with $\mathbf{s}=\left[s_{1}, s_{2}, s_{3}, s_{4}\right]^{T}$ and a virtual channel matrix $\mathbf{H}_{v i}$, resulting in:

$$
\begin{gathered}
\mathbf{H}_{v 1}^{(4)}=\left[\begin{array}{rrrr}
h_{1} & h_{2} & h_{3} & h_{4} \\
-h_{2}^{*} & h_{1}^{*} & -h_{4}^{*} & h_{3}^{*} \\
-h_{3}^{*} & -h_{4}^{*} & h_{1}^{*} & h_{2}^{*} \\
h_{4} & -h_{3} & -h_{2} & h_{1}
\end{array}\right], \text { if } \mathbf{S}_{\mathrm{i}}^{(4)}=\mathbf{S}_{1}^{(4)} ; \\
\mathbf{H}_{v 2}^{(4)}=\left[\begin{array}{rrrr}
-h_{1} & h_{2} & h_{3} & h_{4} \\
-h_{2}^{*} & -h_{1}^{*} & -h_{4}^{*} & h_{3}^{*} \\
-h_{3}^{*} & -h_{4}^{*} & -h_{1}^{*} & h_{2}^{*} \\
h_{4} & -h_{3} & -h_{2} & -h_{1}
\end{array}\right], \text { if } \mathbf{S}_{\mathrm{i}}^{(4)}=\mathbf{S}_{2}^{(4)}
\end{gathered}
$$

Applying matched filtering at the receiver with $\mathbf{H}_{v i}^{(4) H}$ we obtain a Grammian matrix [11] of the following form:

$$
\begin{gathered}
\mathbf{G}_{i}^{(4)}=\mathbf{H}_{v i}^{(4) H} \mathbf{H}_{v i}^{(4)}=\mathbf{H}_{v i}^{(4)} \mathbf{H}_{v i}^{(4) H}=\mathrm{h}^{2}\left[\begin{array}{cc}
\mathbf{I}_{2} & X_{\mathrm{i}} \mathbf{J}_{2} \\
-X_{\mathrm{i}} \mathbf{J}_{2} & \mathbf{I}_{2}
\end{array}\right] \\
(i=1,2) \text { with } \\
\mathbf{I}_{2}=\left[\begin{array}{ll}
1 & 0 \\
0 & 1
\end{array}\right], \mathbf{J}_{2}=\left[\begin{array}{cc}
0 & 1 \\
-1 & 0
\end{array}\right], \\
h^{2}=\left|h_{1}\right|^{2}+\left|h_{2}\right|^{2}+\left|h_{3}\right|^{2}+\left|h_{4}\right|^{2}
\end{gathered}
$$

and an interference parameter $X_{i}$ equal to

$$
\begin{aligned}
& X_{1}=\frac{2 \operatorname{Re}\left(h_{1} h_{4}^{*}-h_{2} h_{3}^{*}\right)}{h^{2}}, \text { if } \mathbf{S}_{1}^{(4)} \text { transmitted } \\
& X_{2}=\frac{2 \operatorname{Re}\left(-h_{1} h_{4}^{*}-h_{2} h_{3}^{*}\right)}{h^{2}}, \text { if } \mathbf{S}_{2}^{(4)} \text { transmitted }
\end{aligned}
$$


It is well known that $\mathbf{G}_{i}^{(4)}$ should approximate a scaled identity-matrix as far as possible to get full diversity and optimum BER performance. This means, $\left|X_{i}\right|$ should be as small as possible. As $\mathbf{G}_{i}^{(4)}$ indicates, our scheme inherently supports full diversity $d=4$, if $X_{i}=0$. Therefore, our strategy is to transmit that code $\mathbf{S}_{1}^{(4)}$ or $\mathbf{S}_{2}^{(4)}$ that minimizes $\left|X_{i}\right|$. As it is assumed that the receiver has full information of the channel state knowing $h_{1}$ to $h_{4}$, the receiver can compute $X_{1}$ and $X_{2}$. With this information the receiver returns the feedback bit $b$ informing the transmitter to select that code block $\mathbf{S}_{i}^{(4)}(i=1,2)$ which leads to the smaller value of $X_{i}$. With this information the transmitter switches between the EACs $\mathbf{S}_{1}^{(4)}$ and $\mathbf{S}_{2}^{(4)}$ such that the resulting $X_{i}$ will correspond to $\min \left(\left|X_{1}\right|,\left|X_{2}\right|\right)$. The statistics of this interference parameter are discussed in [10]. In the simulation it has been assumed that the channel varies slowly such that the delay of the feedback information can be neglected.

\section{B. CAEACs using two feedback bits per code block}

In a quite similar way as discussed in section $\mathrm{A}$ we can switch between four different EACs. In this case, the receiver must send back two bits per code block to support the transmitter to choose the best code matrix for the actual channel realization. The family of our EACs consist of $\mathbf{S}_{1}^{(4)}$ and $\mathbf{S}_{2}^{(4)}$ already defined in Equ.(8) and Equ.(9), and additionally of $\mathbf{S}_{3}^{(4)}, \mathbf{S}_{4}^{(4)}$ defined as:

$$
\begin{aligned}
\mathbf{S}_{3}^{(4)}= & {\left[\begin{array}{rrrr}
j s_{1} & -j s_{2} & s_{3} & s_{4} \\
j s_{2}^{*} & j s_{1}^{*} & s_{4}^{*} & -s_{3}^{*} \\
j s_{3}^{*} & -j s_{4}^{*} & -s_{1}^{*} & -s_{2}^{*} \\
j s_{4} & j s_{3} & -s_{2} & s_{1}
\end{array}\right] } \\
\mathbf{S}_{4}^{(4)}= & {\left[\begin{array}{rrrr}
j s_{1} & j s_{2} & s_{3} & s_{4} \\
j s_{2}^{*} & -j s_{1}^{*} & s_{4}^{*} & -s_{3}^{*} \\
j s_{3}^{*} & j s_{4}^{*} & -s_{1}^{*} & -s_{2}^{*} \\
j s_{4} & -j s_{3} & -s_{2} & s_{1}
\end{array}\right] . }
\end{aligned}
$$

In case of transmitting $\mathbf{S}_{3}^{(4)}$ and $\mathbf{S}_{4}^{(4)}$, the corresponding virtual channel matrices $\mathbf{H}_{v}^{(4)}$ result in:

$$
\begin{gathered}
\mathbf{H}_{v 3}^{(4)}=\left[\begin{array}{rrrr}
j h_{1} & -j h_{2} & h_{3} & h_{4} \\
-j h_{2}^{*} & -j h_{1}^{*} & -h_{4}^{*} & h_{3}^{*} \\
-h_{3}^{*} & -h_{4}^{*} & -j h_{1}^{*} & j h_{2}^{*} \\
h_{4} & -h_{3} & j h_{2} & j h_{1}
\end{array}\right] \\
\mathbf{H}_{v 4}^{(4)}=\left[\begin{array}{rrrr}
j h_{1} & j h_{2} & h_{3} & h_{4} \\
j h_{2}^{*} & -j h_{1}^{*} & -h_{4}^{*} & h_{3}^{*} \\
-h_{3}^{*} & -h_{4}^{*} & -j h_{1}^{*} & -j h_{2}^{*} \\
h_{4} & -h_{3} & -j h_{2} & j h_{1}
\end{array}\right] .
\end{gathered}
$$

The corresponding Grammian matrices $\mathbf{G}_{\mathrm{i}}^{(4)}$ and the channel gain $h$ can be written as in Equ.(12). In case of $\mathbf{S}_{3}^{(4)}$ and $\mathbf{S}_{4}^{(4)}$ the channel dependent interference parameter $X_{i}$ result now in:

$$
\begin{aligned}
& X_{3}=-\frac{2 \operatorname{Im}\left(h_{1} h_{4}^{*}+h_{2} h_{3}^{*}\right)}{h^{2}} \\
& X_{4}=-\frac{2 \operatorname{Im}\left(h_{1} h_{4}^{*}-h_{2} h_{3}^{*}\right)}{h^{2}} .
\end{aligned}
$$

In case of 4 distributed EACs the receiver has to calculate $X_{1}$ to $X_{4}$ and to inform the transmitter which code $\mathbf{S}_{i}^{(4)}$ leads to minimal interference. Then the transmitter switches between the four space-time block codes $\mathbf{S}_{i}^{(4)}$ and chooses that blockcode $\mathbf{S}_{i}^{(4)}$ which leads to an interference parameter $X_{i}$ with minimum absolute value.

\section{CAEACs for eight transmit antennas}

In a similar way, this feedback scheme can be applied to a family of extended Alamouti codes for eight transmit antennas. In case of two EACs the codes $\mathbf{S}_{1}^{(4)}$ given in Equ.(8), $\mathbf{S}_{2}^{(4)}$ in Equ.(9) and $\mathbf{S}_{5}^{(4)}$ given in (5) are used as building blocks for $\mathbf{S}_{1}^{(8)}$ and $\mathbf{S}_{2}^{(8)}$ :

$$
\mathbf{S}_{1}^{(8)}=\left[\begin{array}{rr}
\mathbf{S}_{1}^{(4)} & \mathbf{S}_{5}^{(4)} \\
\mathbf{S}_{5}^{(4) *} & -\mathbf{S}_{1}^{(4) *}
\end{array}\right], \mathbf{S}_{2}^{(8)}=\left[\begin{array}{rr}
\mathbf{S}_{2}^{(4)} & \mathbf{S}_{5}^{(4)} \\
\mathbf{S}_{5}^{(4) *} & -\mathbf{S}_{2}^{(4) *}
\end{array}\right] .
$$

The corresponding virtual channel matrices are:

$$
\mathbf{H}_{v 1}^{(8)}=\left[\begin{array}{rr}
\mathbf{H}_{v 1}^{(4)} & \mathbf{H}_{v 5}^{(4)} \\
-\mathbf{H}_{v 5}^{(4)} & \mathbf{H}_{v 1}^{(4) *}
\end{array}\right], \mathbf{H}_{v 2}^{(8)}=\left[\begin{array}{rr}
\mathbf{H}_{v 2}^{(4)} & \mathbf{H}_{v 5}^{(4)} \\
-\mathbf{H}_{v 5}^{(4)} & \mathbf{H}_{v 2}^{(4) *}
\end{array}\right]
$$

For $\mathbf{G}_{i}^{(8)}$ we get in both cases:

$\mathbf{G}_{i}^{(8)}=\mathbf{H}_{v i}^{(8) H} \mathbf{H}_{v i}^{(8)}=h^{2}\left[\begin{array}{rrrr}\mathbf{I}_{2} & U_{\mathrm{i}} \mathbf{J}_{2} & -V_{i} \mathbf{J}_{2} & Y_{i} \mathbf{I}_{2} \\ -U_{\mathrm{i}} \mathbf{J}_{2} & \mathbf{I}_{2} & -Y_{i} \mathbf{I}_{2} & -V_{i} \mathbf{J}_{2} \\ V_{i} \mathbf{J}_{2} & -Y_{i} \mathbf{I}_{2} & \mathbf{I}_{2} & U_{\mathrm{i}} \mathbf{J}_{2} \\ Y_{i} \mathbf{I}_{2} & V_{i} \mathbf{J}_{2} & -U_{\mathrm{i}} \mathbf{J}_{2} & \mathbf{I}_{2}\end{array}\right]$,

where the channel dependent interference parameters $U_{i}, Y_{i}$ and $V_{i}$ can be calculated as:

$$
\begin{aligned}
& U_{1}=2 \operatorname{Re}\left(h_{1} h_{4}^{*}-h_{2} h_{3}^{*}+h_{5} h_{8}^{*}-h_{6} h_{7}^{*}\right) / h^{2} \\
& Y_{1}=2 \operatorname{Re}\left(h_{1} h_{7}^{*}-h_{3} h_{5}^{*}+h_{2} h_{8}^{*}-h_{4} h_{6}^{*}\right) / h^{2} \\
& V_{1}=2 \operatorname{Re}\left(-h_{1} h_{6}^{*}+h_{2} h_{5}^{*}+h_{4} h_{7}^{*}-h_{3} h_{8}^{*}\right) / h^{2}
\end{aligned}
$$

if $\mathbf{S}_{1}^{(8)}$ is sent, and

$$
\begin{aligned}
& U_{2}=2 \operatorname{Re}\left(-h_{1} h_{4}^{*}-h_{2} h_{3}^{*}+h_{5} h_{8}^{*}-h_{6} h_{7}^{*}\right) / h^{2} \\
& Y_{2}=2 \operatorname{Re}\left(-h_{1} h_{7}^{*}-h_{3} h_{5}^{*}+h_{2} h_{8}^{*}-h_{4} h_{6}^{*}\right) / h^{2} \\
& V_{2}=2 \operatorname{Re}\left(h_{1} h_{6}^{*}+h_{2} h_{5}^{*}+h_{4} h_{7}^{*}-h_{3} h_{8}^{*}\right) / h^{2}
\end{aligned}
$$

if $\mathbf{S}_{2}^{(8)}$ is sent. $\mathbf{I}_{2}$ and $\mathbf{J}_{2}$ are defined in Equ.(12).

In case of 4 EACs the transmitter can switch between $\mathbf{S}_{1}^{(8)}$, $\mathbf{S}_{2}^{(8)}$, given in Equ.(19), and $\mathbf{S}_{3}^{(8)}$ and $\mathbf{S}_{4}^{(8)}$ defined as:

$$
\mathbf{S}_{3}^{(8)}=\left[\begin{array}{cc}
\mathbf{S}_{3}^{(4)} & \mathbf{S}_{5}^{(4)} \\
\mathbf{S}_{5}^{(4) *} & -\mathbf{S}_{3}^{(4) *}
\end{array}\right], \mathbf{S}_{4}^{(8)}=\left[\begin{array}{cc}
\mathbf{S}_{4}^{(4)} & \mathbf{S}_{5}^{(4)} \\
\mathbf{S}_{5}^{(4) *} & -\mathbf{S}_{4}^{(4) *}
\end{array}\right]
$$

The corresponding virtual channels can be calculated as:

$$
\mathbf{H}_{v 3}^{(8)}=\left[\begin{array}{rr}
\mathbf{H}_{v 3}^{(4)} & \mathbf{H}_{v 5}^{(4)} \\
-\mathbf{H}_{v 5}^{(4) *} & \mathbf{H}_{v 3}^{(4) *}
\end{array}\right], \mathbf{H}_{v 4}^{(8)}=\left[\begin{array}{rr}
\mathbf{H}_{v 4}^{(4)} & \mathbf{H}_{v 5}^{(4)} \\
-\mathbf{H}_{v 5}^{(4) *} & \mathbf{H}_{v 4}^{(4) *}
\end{array}\right]
$$

with

$$
\begin{aligned}
& U_{3}=\left(-2 \operatorname{Im}\left(h_{1} h_{4}^{*}+h_{2} h_{3}^{*}\right)+2 \operatorname{Re}\left(h_{5} h_{8}^{*}-h_{6} h_{7}^{*}\right)\right) / h^{2} \\
& Y_{3}=\left(-2 \operatorname{Im}\left(h_{1} h_{7}^{*}-h_{2} h_{8}^{*}\right)+2 \operatorname{Re}\left(-h_{3} h_{5}^{*}-h_{4} h_{6}^{*}\right)\right) / h^{2} \\
& V_{3}=\left(-2 \operatorname{Im}\left(-h_{1} h_{6}^{*}-h_{2} h_{5}^{*}\right)+2 \operatorname{Re}\left(h_{4} h_{7}^{*}-h_{3} h_{8}^{*}\right)\right) / h^{2},
\end{aligned}
$$


if $\mathbf{S}_{3}^{(8)}$ is sent, and in case $\mathbf{S}_{4}^{(8)}$ is sent:

$$
\begin{aligned}
U_{4} & =\left(-2 \operatorname{Im}\left(h_{1} h_{4}^{*}-h_{2} h_{3}^{*}\right)+2 \operatorname{Re}\left(h_{5} h_{8}^{*}-h_{6} h_{7}^{*}\right)\right) / h^{2} \\
Y_{4} & =\left(-2 \operatorname{Im}\left(h_{1} h_{7}^{*}+h_{2} h_{8}^{*}\right)+2 \operatorname{Re}\left(-h_{3} h_{5}^{*}-h_{4} h_{6}^{*}\right)\right) / h^{2} \\
V_{4} & =\left(-2 \operatorname{Im}\left(-h_{1} h_{6}^{*}+h_{2} h_{5}^{*}\right)+2 \operatorname{Re}\left(h_{4} h_{7}^{*}-h_{3} h_{8}^{*}\right)\right) / h^{2} .
\end{aligned}
$$

In case of eight transmit antennas we have three channel dependent parameters $U_{i}, Y_{i}$ and $V_{i}$ causing cross-signal interference. To obtain full diversity and optimum BER performance, all three parameter $U_{i}, Y_{i}$ and $V_{i}$ should be as small as possible. There are different ways to approach this minimum. The best method we found, relates to minimizing the condition number of the Grammian $\mathbf{G}$ defined as the ratio of the largest to the smallest eigenvalue $\left(\kappa=\operatorname{cond}(\mathbf{G})=\frac{\max \left(\lambda_{\mathbf{G}}\right)}{\min \left(\lambda_{\mathbf{G}}\right)}\right)$. In [10] it is shown that the eigenvalues of $\mathbf{G}$ can easily be computed given the triple $\{U, Y, V\}$.

\section{Simulations Results}

In our simulations, we have used a QPSK signal constellation. The Rayleigh fading channel has been kept constant during the transmission of each code block but has faded independently between successive blocks. At the receiver side, we have implemented a zero forcing (ZF) receiver and a maximum likelihood (ML) receiver. The resulting curves are compared with an ideal two, four and eight path diversity transmission (no cross interference). The BER results have been averaged over 2,048 QPSK information symbols and 10,000 realizations of the i.i.d. channel matrix.

Fig. 2 and Fig. 3 show the BER as a function of $E_{b} / N_{0}$ applying the ZF and ML receiver, respectively, utilizing 4 transmit antennas. The resulting BER for the $(8 \times 1)$ scheme for both receiver types are depicted in Fig. 4. Obviously a substantial improvement of the BER can be achieved by returning only a small amount of channel information to the transmitter. Typically just one bit of feedback information improves the diversity considerably, while two bits nearly preserve full diversity. In the $(8 \times 1)$ scheme an ML receiver achieves the full diversity of $d=8$ with only one feed back bit.

Applying the Kronecker Model [9], we model fading correlation by the following channel matrix:

$$
\mathbf{H}=\frac{1}{\sqrt{\operatorname{tr}\left(\mathbf{R}_{T}\right)}} \mathbf{R}_{R}^{\frac{1}{2}} \mathbf{W} \mathbf{R}_{T}^{\frac{1}{2}}
$$

where $\mathbf{R}_{R}=\mathrm{E}\left\{\mathbf{H H}^{H}\right\}$ is the $n_{R} \times n_{R}$ receive correlation matrix, and $\mathbf{R}_{T}=\mathrm{E}\left\{\mathbf{H}^{H} \mathbf{H}\right\}$ is the $n_{T} \times n_{T}$ transmit correlation matrix. The term $\mathbf{W}$ describes a random $n_{R} \times n_{T}$ matrix with independent complex-valued elements with zero mean and unit variance. The elements of $\mathbf{W}$ are assumed to be Gaussian distributed. The normalization coefficient

$$
\operatorname{tr}\left(\mathbf{R}_{T}\right)=\operatorname{tr}\left(\mathbf{R}_{R}\right)=\mathrm{E}\left\{\sum_{i=1}^{n_{R}} \sum_{j=1}^{n_{T}}\left|h_{i j}\right|^{2}\right\}
$$

can be interpreted as the channel's total power transmission factor. We simulated the $(4 \times 1)$ MIMO system with $\mathbf{R}_{R}=\mathbf{I}$,

$$
\mathbf{R}_{\mathrm{T}}=\left[\begin{array}{llll}
1 & \rho & \rho^{2} & \rho^{3} \\
\rho & 1 & \rho & \rho^{2} \\
\rho^{2} & \rho & 1 & \rho \\
\rho^{3} & \rho^{2} & \rho & 1
\end{array}\right]
$$

assuming antenna elements correlated by factors of $\rho=$ $\{0.5,0.75,0.95\}$. Figures 5-8 show the simulation results for $\mathrm{ZF}$ and ML receiver when the transmitter switches between 2 and 4 EACs. It turns out that for small correlation up to $\rho=$ 0.5 the feedback information still gives strong improvement as in the i.i.d case. However with larger correlation the feedback information does not improve the transmission very much.

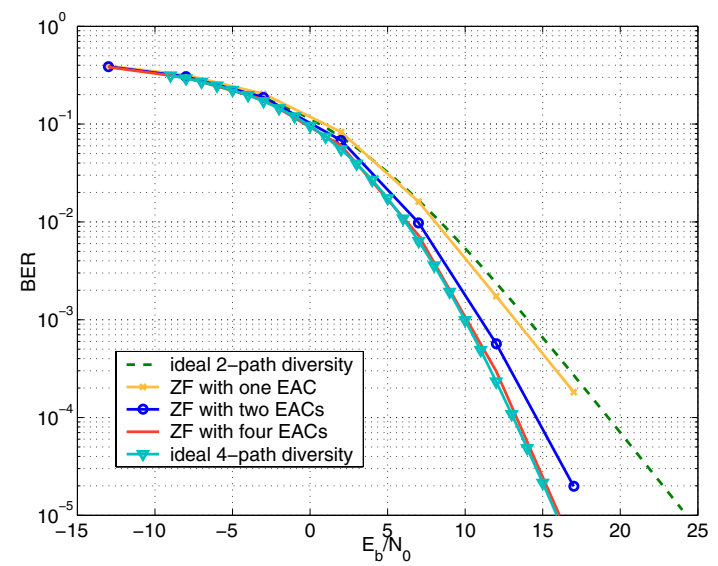

Fig. 2. BER of $(4 \times 1)$ extended Alamouti scheme with feedback and ZF Receiver

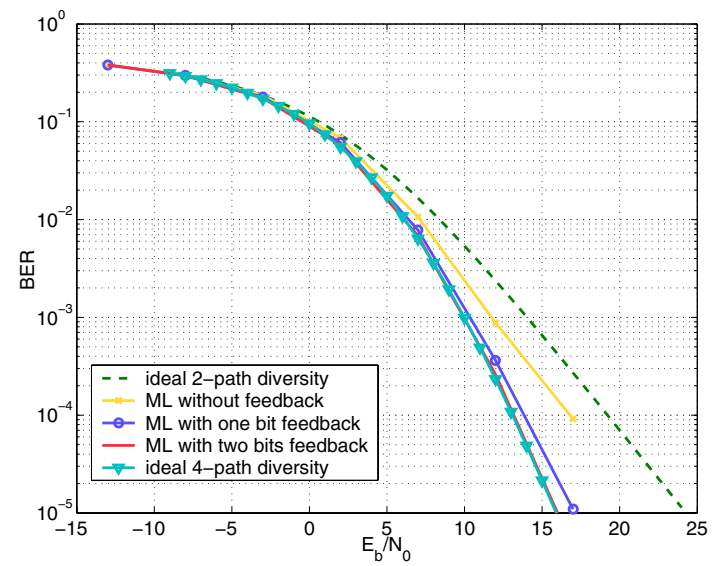

Fig. 3. BER of $(4 \times 1)$ extended Alamouti scheme with feedback and ML Receiver

\section{Conclusions}

In this work, we present a MIMO-system, where the transmitter switches between 2 and 4 EACs depending on an instantaneous channel parameter fed back from the receiver to the transmitter. We have shown that this simple transmission scheme improves diversity and BER compared to the case of an open loop transmission system as far as the channel correlation is not to high. The simple $\mathrm{ZF}$ receiver performs essentially as well as the ML receiver. 


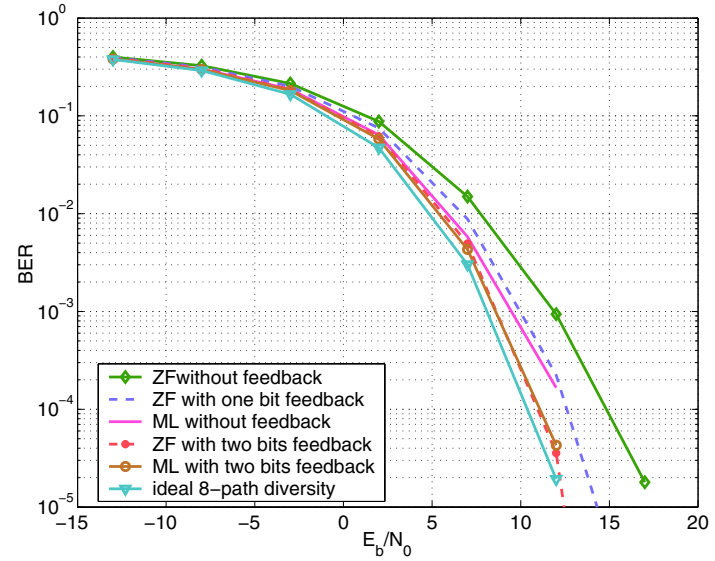

Fig. 4. BER of $(8 \times 1)$ extended Alamouti scheme with feedback, ZF and ML Receiver

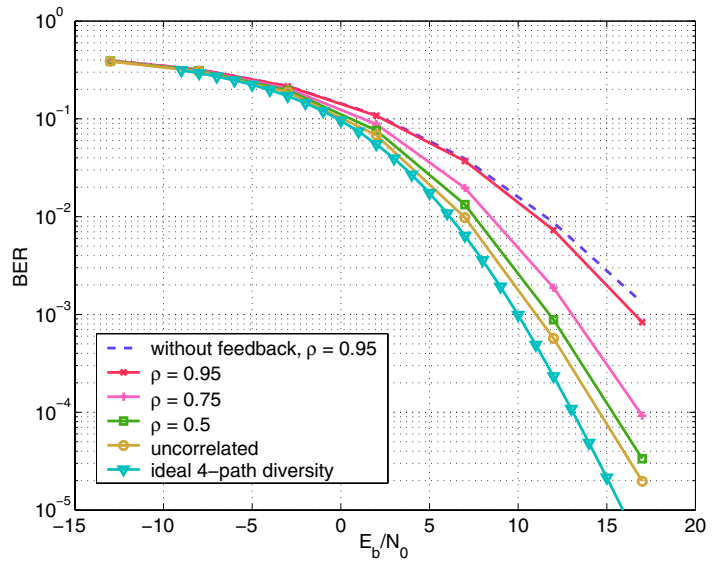

Fig. 5. BER of $(4 \times 1)$ extended Alamouti scheme with one bit feedback, ZF Receiver and fading correlation

\section{REFERENCES}

[1] S.M Alamouti "A Simple Diversity Technique for Wireless Communications," IEEE J. Sel. Ar. Comm., vol.16, no.8, pp. 1451-1458, Oct. 1998.

[2] V.Tarokh, H. Jafarkhani and A.R. Calderbank, "Space-Time block codes from orthogonal designs," IEEE Trans.Inf. Theory, vol.45, pp. 1456-1467, July 1999.

[3] H. Jafarkhani, "A quasi orthogonal space-time block code," IEEE Trans. Comm., vol. 49, pp. 1-4, Jan. 2001.

[4] J.Akhtar, D.Gesbert, J.Akhtar, D.Gesbert "Partial Feedback Based Orthogonal Block Coding," Proceedings IEEE Vehicular Technology Conference, VTC 2003-Spring, vol.1, pp.287-291, April, 2003.

[5] G.Jongren, M. Skoglund, B. Ottersten, "Combining beamforming and orthogonal space-time block coding0", IEEE Trans.Inf. Theory, vol.48, pp. 611-627, March 2002.

[6] G.Jongren, M. Skoglund, "Utilizing quantized feedback information in orthogonal space-time block coding". in Proc. IEEE Globcom, vol. 2, pp. 995-999, Nov.-Dec. 2000.

[7] E.G. Larsson, G. Ganesan, P. Stoica, W.-H. Wong, "On the performance of the orthogonal space-time block coding with quantized feedabck", IEEE Communication Letters. vol. 6, pp. 487-469, Nov. 2002.

[8] M.Kiessling, J.Speidel, "Performance analysis of MIMO maximum likelihood receivers with channel correlation, colored Gaussian noise and linear prefiltering," Proc. of ICC03, pp. 3026-3030, May 2003.

[9] M.T.Ivrlac, W.Utschick, J.A.Nosek, "Fading Correlations in Wireless MIMO Communication Systems", IEEE Journal on Selected Areas in Communications, vol. 21, No. 5, pp. 819-828, June 2003

[10] C.F.Mecklenbräuker, M.Rupp "Flexible Space-Time Block Codes for Trading Quality of Service against Data Rate in MIMO UMTS", EURASIP Journal on Applied Signal Processing, Special Issue on MIMO Communication and Signal Processing, to appear 2004.

[11] M.Rupp, C.F.Mecklenbräuker, "On Extended Alamouti Schemes for Space-Time Coding, " Proc. WPMC'02, Honolulu, pp. 115-119, Oct. 2002.

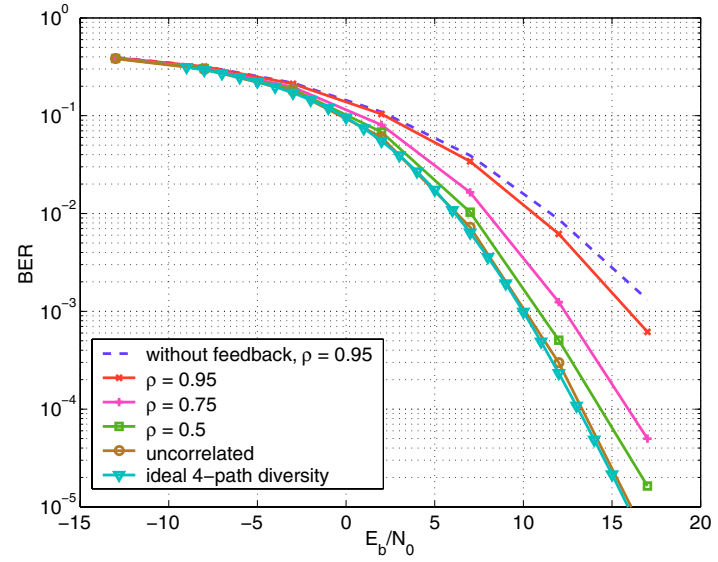

Fig. 6. BER of $(4 \times 1)$ extended Alamouti scheme with two bits feedback, ZF Receiver and fading correlation

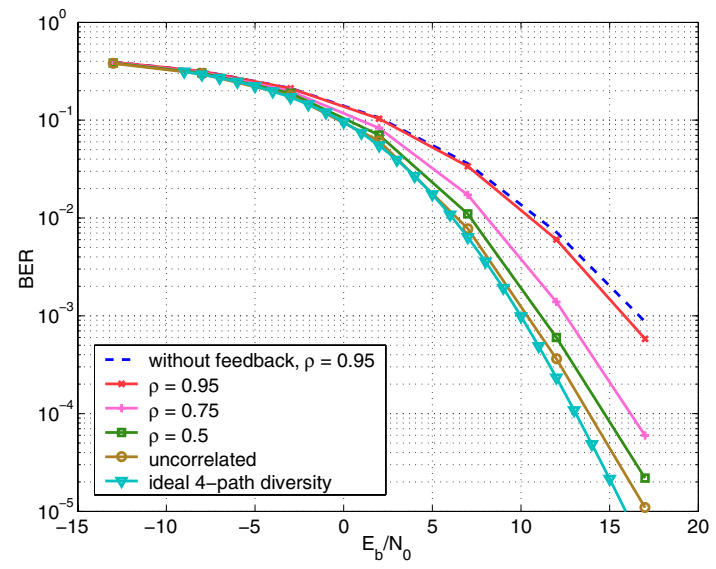

Fig. 7. BER of $(4 \times 1)$ extended Alamouti scheme with one bit feedback, ML Receiver and fading correlation

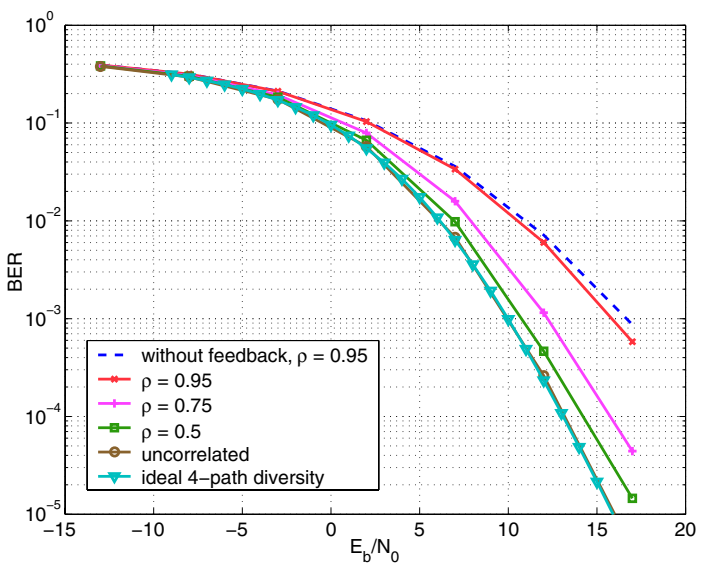

Fig. 8. BER of $(4 \times 1)$ extended Alamouti scheme with two bits feedback, ML Receiver and fading correlation 